\title{
The case of the critically ill fisherman
}

\author{
Leah Nemiroff MD, MSc', Ahmed Ghaly MD², David J.M. Haldane MD³, \\ Tobias Witter MD \\ I Department of Medicine, Dalhousie University, Halifax, NS, Canada \\ 2 Department of Medicine, Division of Infectious Diseases, Dalhousie University, Halifax, NS, Canada \\ 3 Department of Pathology, Clinical Director Provincial Public Health Laboratory Network of Nova \\ Scotia, Director of Special Pathogens NSHA, Dalhousie University, Halifax, NS, Canada \\ 4 Departments of Anesthesia and Medicine, Division of Critical Care, Dalhousie University, Halifax, NS, \\ Canada
}

$\mathrm{C}$

ommercial fishermen are considered to have one of the most dangerous professions in the world, with an estimated mortality rate of up to 45.8 in 100,000 fishermen in Atlantic Canada per year. ${ }^{1}$ In addition to working in extreme weather conditions, experiencing physical discomfort, psychological stress and physical injury, exposure to infectious pathogens can also occur. This case describes the clinical course of a young, immunocompetent, fisherman with refractory sepsis and multiple organ failure caused by Erysipelothrix rhusiopathiae, a rare but well-described occupational pathogen associated with farming and fisheries. Erysipelothrix infection usually causes cutaneous lesions or, more rarely, bacteremia with endocarditis in immunocompromised individuals. This case illustrates the importance of a detailed history in the diagnosis and management of patients with atypical presentations.

\section{Case Summary}

A 27-year-old, previously healthy man, presented to his local emergency department with a 24-hour history of nausea, vomiting, and worsening respiratory distress. His only relevant history was consumption of 2-4 alcoholic drinks per day. On admission, he was afebrile, but hypotensive (systolic blood pressure 90-100 $\mathrm{mmHg})$, tachycardic (120 bpm), and required $100 \%$ FiO2 to maintain an SpO2 greater than 90\%. His bloodwork indicated severe sepsis with fulminant liver and renal failure, including a lactate of $27.3 \mathrm{mmol} / \mathrm{L}$, INR 5.6, glucose $1.4 \mathrm{mmol} / \mathrm{L}$, total bilirubin 140.9 $\mu \mathrm{mol} / \mathrm{L}$, AST 28,014 U/L, ALT 11,497 U/L, creatinine $426 \mu \mathrm{mol} / \mathrm{L}$, white blood cell count $13.2 \times 10^{9} / \mathrm{L}$, and platelets $23 \times 10^{9} / \mathrm{L}$.

The patient was intubated and transferred to a tertiary care center Intensive Care Unit. He required aggressive fluid resuscitation and inotropic support for refractory hypotension. He was treated empirically with a broad-spectrum antibiotic (piperacillin/ tazobactam 2.25g IV every 6 hours) for septic shock of unknown etiology. Aerobic and anaerobic blood cultures drawn on day one of his hospitalization grew Gram-positive rods, later identified as Erysipelothrix rhusiopathiae (susceptible to penicillins, cephalospo- rins, quinolones, clindamycin, and erythromycin, but resistant to vancomycin) and Methicillin Sensitive Staphylococcus aureus (MSSA). Repeat blood cultures on day 4 continued to grow only E. rhusiopathiae. A transthoracic echocardiogram was done with high quality images that showed normal valves with no evidence of endocarditis. Subsequent blood cultures, taken on day 8 and 9 , were sterile. Viral serology was negative, including hepatitis $\mathrm{B}$, hepatitis $\mathrm{C}$, and HIV.

A detailed history with collateral revealed that the patient had been self-medicating for musculoskeletal pain with extra-strength acetaminophen and ibuprofen. His liver failure was attributed to a combination of acetaminophen overdose and hypoperfusion in the context of septic shock. Further inquiry elicited that he was a fisherman by trade and had a work-related injury (fish bite) on his left hand while working two weeks prior. Examination of his left hand showed a cutaneous lesion that appeared consistent with an erysipeloid infection.

Unfortunately, despite appropriate therapy, the patient died on day 14 of his hospitalization from multiple organ system failure secondary to refractory septic shock.

\section{Discussion}

Erysipelothrix rhusiopathiae is a Gram-positive, non-acid-fast, non-spore forming, facultatively anaerobic, rod-shaped bacterium. It is known to be highly susceptible to penicillins, cephalosporins and clindamycin, but is one of few Gram-positive organisms resistant to vancomycin. ${ }^{2}$

E. rhusiopathiae is a widespread organism in nature and is a pathogen or a commensal in many wild and domestic mammals, birds, crustaceans, and fish., ${ }^{2,3}$ The organism was first identified by Robert Koch in 1878 and later established as a human pathogen by Friedrich Rosenbach in $1909 .^{3}$ Swine erysipelas due to E. rhusiopathiae is one of the most well-known and documented disease presentations in animals, as outbreaks can have devastating consequences to herd health and farming productivity. ${ }^{4}$ E. rhusiopathiae has been identified as a cause of superficial and invasive 
soft tissue infection in humans following marine injuries and exposures, and outbreaks of erysipeloid have been associated with occupational exposure to fish and crustaceans. ${ }^{3,5,6}$ This organism survives and grows on the exterior mucoid slime of fish and other marine creatures without causing disease in the animals themselves. ${ }^{2}$

Three main forms of human E. rhusiopathiae infection have been described. The most common is erysipeloid, a localized cellulitis usually found on the fingers or hands of a patient due to contact with infected animals or their products. This is usually unaccompanied by fever or systemic symptoms. There is also a more generalized cutaneous form with systemic features including fever and arthralgias, in the setting of negative blood cultures. ${ }^{3}$ Finally, E. rhusiopathiae infection can present as bacteremia, commonly associated with native valve endocarditis. ${ }^{7}$ In 49 reported cases of E. rhusiopathiae bacteremia over 15 years, the incidence of endocarditis was $90 \%$, with $60 \%$ being on previously normal heart valves. ${ }^{3}$ These rates are much higher than many other organisms typically associated with endocarditis. ${ }^{3}$

There are an increasing number of reports of patients with E. rhusiopathiae bacteremia with no evidence of endocarditis, although this is usually in immunocompromised hosts. ${ }^{8}$ In patients who do present with E. rhusiopathiae bacteremia, septic shock is an extremely rare occurrence. ${ }^{9,10}$ Sinclair et al. describe a rapidly progressive sepsis secondary to E. rhusiopathiae bacteremia in an immunocompromised patient, that may have been acquired from a fishing wire injury. ${ }^{6}$ Another case report by Surrun et al. reported a diabetic patient without endocarditis with E. rhusiopathiae bacteremia that initially presented with septic shock. Her initial insult appeared to be a localized cellulitis on her foot from injury while wearing slippers at a fish market. ${ }^{10}$

Interestingly, infection with $E$. rhusiopathiae in immunocompetent patients has been associated with alcohol abuse. ${ }^{11,12}$ A study by Reboli \& Farrar showed that among 49 bacteremic patients, 33\% with endocarditis also reported alcohol abuse. ${ }^{3}$ It was also the only identifiable risk factor in an E. rhusiopathiae infection in an immunocompetent patient without endocarditis described by Fakoya et al. ${ }^{12}$ Our patient was previously very healthy aside from moderate to severe alcohol intake. As such, his history of alcohol use may have made him more vulnerable to $E$. rhusiopathiae infection than the general population with similar occupational exposures. The physiologic stress of his multifactorial liver injury may have then resulted in a relative immunocompromised state, enabling a subclinical infection to progress to sepsis.

\section{Conclusion}

E. rhusiopathiae infection associated with rapidly progressive sepsis and septic shock in a previously immunocompetent patient without endocarditis is a rare clinical event. This case emphasizes the importance of a thorough occupational and social history in patients with sepsis of unknown etiology to demonstrate risk factors for unusual pathogens. A high index of suspicion should be raised in patients with known occupational exposures and with Gram-positive rods in blood cultures failing to respond to vancomycin., ${ }^{2,11}$ Moreover, since most human E. rhusiopathiae infections result from occupational exposures, it is important to emphasize awareness and safe work practices in vulnerable populations.

\section{References}

1. Hasselback P, Neutel I. Risk for commercial fishing deaths in Canadian atlantic provinces. Br J Ind Med 1990;47:498-501.

2. Brooke CJ, Riley TV. Erysipelothrix rhusiopathiae: bacteriology, epidemiology and clinical manifestations of an occupational pathogen. J Med Microbiol 1999;48(9):789-799.

3. Reboli A, Farrar WE. Erysipelothrix rhusiopathiae: an occupational pathogen. Clin Microbiol Rev 1989;2(4):354-359.

4. Wang Q, Chang BJ, Riley TV. Erysipelothrix rhusiopathiae. Vet Microbiol 2010;140(3-4):405-417.

5. Diaz JH. Emerging causes of superficial and invasive infections following marine injuries and exposures. J La State Med Soc 2014;166(3):103-108.

6. Sinclair M, Hawkins A, Testro A. Something fishy: an unusual Erysipelothrix rhusiopathiae infection in an immunocompromised individual. BMJ Case Rep 2013; <http://casereports.bmj. com> (Sept 24, 2014).

7. Kaya S, Gencalioglu E, Yildrim SS, Altun G, Yilmaz G, Koksal I. Native valve endocarditis cause by Erysipelothrix rhusiopathiae in an immunocompetent individual. J Med Microbiol 2013;62(pt12):1911-1913.

8. Drekonja DM. Erysipelothrix bacteremia without endocarditis: rare event or under-reported occurrence? Diagn Microbiol Infect Dis 2013;77(3):280-281.

9. Ognibene FP, Cunnion RE, Gill V, Ambrus J, Fauci AS, Parillo JE. Erysipelothrix rhusiopathiae bacteremia presenting as septic shock. Am J Med 1985;78:861-864.

10. Surrun SK, Jaufeerally FR, Sim HC. Erysipelothrix rhuseopathiae septicaemia with prolonged hypotension: a case report. AAMS 2008;37(3):251-252.

11. Schuster MG, Brennan PJ, Edelstein P. Persistent bacteremia with Erysipelothrix rhusiopathiae in a hospitalized patient. Clin Infect Dis 1993;17:783-784.

12. Fakoya A, Bendall RP, Churchill DR, Doherty JF, Ridgway GL. Erysipelothrix rhusiopathiae bacteraemia in a patient without endocarditis. J Infect 1994;30(2):180-181. 